\title{
Elementary concepts in chaos and turbulence
}

\author{
RAMAKRISHNA RAMASWAMY \\ Tata Institute of Fundamental Research, Homi Bhabha Road, Colaba, Bombay 400005, India
}

\begin{abstract}
The phenomenology of aperiodic or chaotic behaviour is described with reference to simple theoretical models and experiments. A brief description is given of the current understanding of how irregular dynamical motions can arise.
\end{abstract}

Keywords. Chaos; nonlinear dynamics; turbulence.

\section{Introduction}

In the past few years, an intense effort has been devoted towards the detailed study of intrinsically random phenomena. Random phenomena are plentiful; in this talk, I will attempt to give a brief introduction to the rather simple concepts that at present underlie a basic understanding of chaos or irregularity, and the onset of turbulence. Many of the ideas are novel and interesting; whether or not these concepts are directly applicable in the context of mechanical behaviour as discussed in this Meeting is not entirely clear, but that is more a problem of application. This talk will be primarily phenomenological and qualitative without much emphasis on the mathematics (although much of the insight in this field really comes from the mathematics). Rather than give explicit references through the text, I prefer to list in the bibliography several articles that discuss these concepts in greater detail.

It is of interest to examine families of (nonlinear) systems rather than particular cases, as this type of study is apt to reveal more of the general behaviour. Typically, such systems can be parametrized by some physical variable such as temperature, pressure, applied voltage, etc. In many situations, the following general pattern has been seen to occur as a function of the relevant control parameter:

Smooth behaviour $\longrightarrow$ transitional $\longrightarrow$ range $\stackrel{\text { erratic }}{\text { behaviour }}$

periodic $\longrightarrow$ ? $\longrightarrow$ aperiodic

A few examples are given below.

(1) Heart beats are possibly the most familiar instance of regular, periodic behaviour. As a function of applied stress (for example during physical exertion), we can easily observe the period changing as the heart beats faster. When this exertion is now coupled with oxygen availability, when one is climbing a mountain, say, the period can change drastically, and in fact may become quite erratic. This has unfortunate physiological effects (death is likely), and emphasises the importance of a transition from regular to irregular behaviour under the variation of a control parameter.

(2) A less familiar example occurs in the study of animal populations. This assumes 
importance in livestock farming or fishery management, where it is necessary to know the behaviour of the population as a function of time, typically from generation to generation. One can in fact write fairly simple equations (of the difference, differential, or differential-delay type) to describe the population, where the control parameters are the food supply and the reproductive ability. These simple equations can give rise to totally chaotic behaviour for some values of these parameters.

(3) An example that I will discuss later on has to do with the onset of turbulence. In the classic Rayléigh-Benard experiment, when a liquid is heated, convective motion is established within the liquid. As the rate of heating is increased, the motion can become quite irregular, leading eventually to the full scale turbulence which is seen in the boiling liquid.

(4) It is possible that in a material under stress, this kind of behaviour is present as well, as may be deduced from the equations that model serrated yielding, for some values of the applied strain.

There are several more examples that one could cite. What is important, however, is that chaos is a dynamical phenomenon, and is abundant, in that it occurs frequently in nonlinear situations. (Nonlinearity of the dynamical system is a necessary condition for chaos, but is not a sufficient one.)

\section{Dynamics}

We define a dynamical system in terms of one independent variable, such as time, $t$, one or more dependent variables, $x_{i}(t)$, and an external (control) parameter, $\mu$. One requires a prescription to determine the evolution of the dependent variables. This is done via a system of differential equations,

$$
\frac{\mathrm{d}}{\mathrm{d} t} x_{i}(t)=F_{\mu}(\mathbf{x}(t))
$$

or difference equations,

$$
x_{i}(j+1)=F_{\mu}(\mathbf{x}(j)) .
$$

The index $j$ plays the role of a discrete time in the latter case. The parametric dependence is contained in $F_{\mu}$. Given these and a set of initial conditions, it is straightforward to obtain the time evolution of the dependent variables. This defines an orbit, and it is the behaviour of these orbits as a function of time that describes the dynamics. It is customary to examine the dynamics in the phase space of the variables $x_{i}(t), i=1,2 \ldots$

We can simply define regular behaviour as motion, $x_{i}(t)$, which is predictable. That is, the dynamical variables are periodic functions of time, or combinations of periodic functions, i.e., quasiperiodic functions. Irregular or chaotic motion is, in contrast, unpredictable, and therefore is given by an aperiodic function (equivalently, of infinite period). It is important to emphasize that these motions are discussed entirely in terms of deterministic evolution via (1) or (2). Chaotic motions arise independent of random noise or fluctuations. In fact, it is customary to label such intrinsic erratic behaviour as self-generated chaos.

The notions of regular and chaotic motion can be quantified in several ways. A direct and rather obvious means is through the spectral or Fourier analysis of the orbits. 
Regular motion leads naturally to a discrete Fourier spectrum, since the motion is either periodic or quasiperiodic. The spectrum for chaotic motion must necessarily be continuous owing to the aperiodicity (i.e., any and all frequencies-in particular, zerofrequency). Another means of distinction is based on the Liapunov instability of orbits. The euclidean distance between regular orbits can grow linearly; whereas, between chaotic orbits, it grows exponentially. It is also possible to examine the orbits themselves, to get an idea of how they develop in phase space. When there are $n$ dependent variables, corresponding to $m$ degrees of freedom, the phase space is of dimension $n\left(x_{i}(t) ; i=1,2, \ldots, n\right)$. Examining the motion in this $n$-dimensional space is a difficult task. One can, however, essentially accomplish this by examining the Poincare surface-of-section. This works best when the number of degrees of freedom is small, $m=2$ or 3 . To construct a surface of section, one chooses a particular plane in the phase space, and locates the point of intersection each time the orbit passes through this plane. If a given orbit is examined for a sufficiently long time, there are several points of intersection, and these give an idea of the geometry of the orbit. For regular motions, the points of the Poincare section lie on smooth curves, whereas for chaotic motions, these points are scattered apparently randomly in the plane (independent of which particular plane is chosen).

In discussing dynamical systems, it is useful to distinguish between conservative systems (where the volume of phase space is constant in time) and non conservative systems (where this volume changes with time). The behaviour is somewhat different in the two cases. In most physical situations one encounters dissipative, nonconservative systems, owing to the presence of frictional forces, and in that sense these systems are more important. It is however necessary for completeness to describe in brief the behaviour of conservative systems.

\subsection{Conservative (Hamiltonian) systems}

The most common examples of conservative systems are Hamiltonian systems, wherein one can define a Hamiltonian function, $H(\mathbf{x})$. As general dynamical systems, these have $n=2 m$ dependent variables for $m$ degrees of freedom. Thus, the phase space is of dimension $2 m ; m$ of these variables are 'coordinates', and $m$ are their conjugate 'momenta'. Evolution is governed by Hamilton's equations. (In the discrete case, the requirement of constant phase space volume is replaced by the condition that the Jacobian of the transformation in (2) have the value \pm 1 ). One can further separate such systems into two classes: integral systems and nonintegral systems. In the former class, it is possible to make a canonical transformation such that $H(x)$ is given in terms of $m$ (in general, new) 'momentum' variables alone. These are constants of the motion, which is then constrained to lie on well-defined geometrical objects, namely, $m$-dimensional tori in the phase space of dimension $2 \mathrm{~m}$. Thus the motion is always regular. We can regard nonintegrable Hamiltonians as arising from integrable ones by the addition of perturbation terms (depending on $\mu$ ), such that $H$ can no longer be expressed as a function of $m$ momentum-like variables alone. There is then no guarantee that the motion will lie on $m$-dimensional tori. The powerful KAM theorem applies in such cases. Loosely speaking, this theorem states that, depending on the magnitude of the perturbation, some of the tori that exist in the integrable case get distorted, and some of them are destroyed. When the tori are merely distorted, the motion still remains regular; but when they are destroyed, the orbits are free to wander over all of phase 
space, and can therefore become highly irregular and chaotic. The phenomenology of Hamiltonian chaos is in fact extremely bizzare and involved; this erratic behaviour basically arises from the complicated interactions between internal resonances. The foregoing is a cursory description of the bare essentials of the matter. As a simple example, consider the discrete map

$$
\begin{aligned}
& x_{i+1}=\mu x_{i}-\left(y_{i}-x_{i}^{2}\right)(1-\mu)^{1 / 2}, \\
& y_{i+1}=x_{i}(1-\mu)^{1 / 2}+\left(y_{i}-x_{i}^{2}\right) \mu .
\end{aligned}
$$

This roughly models the Poincaré surface of section of an $m=2$ degree of freedom Hamiltonian system. Orbits are constructed by iterating points in the xy plane under the above transformations. Depending on where one starts, successive points in figure 1 can lie on a smooth curve (A) or on a set of smooth curves (B)-these correspond to regular motion on tori; or else the points can be scattered all over the plane $(C)$-this is a chaotic orbit and is not confined to any simple geometrical object. In general, the motion can be extremely complicated. This is both typical, in the sense that most nonlinear Hamiltonian systems are likely to be nonintegrable, and pathological, in the sense that for any nonzero value of $\mu$, a nonintegrable Hamiltonian will exhibit some chaotic motions.

\subsection{Dissipative systems}

As already mentioned, dissipative systems are more common, and thus more pertinent. A typical example is provided by the forced-beam problem. A beam of length $b$ is

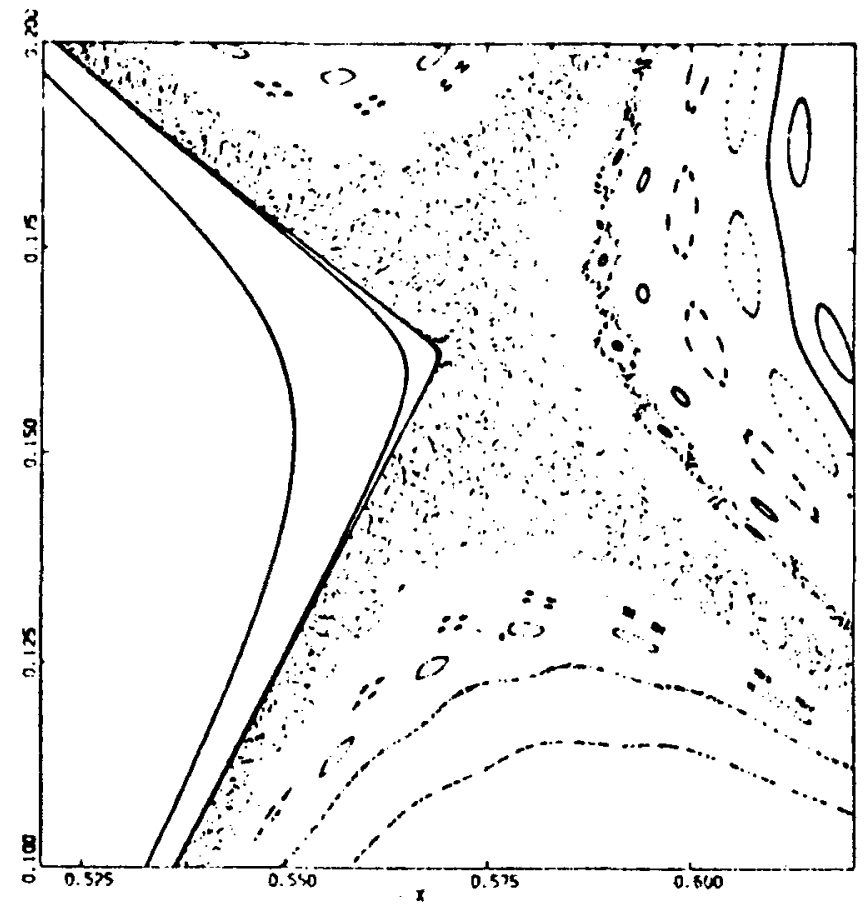

Figure 1. Orbits in the $x y$ plane induced by the discrete transformation of equations (3)-(4). $A$ and $B$ are examples of regular motion, while the set of points $C$ is typical of the Poincaré section of a chaotic orbit. 
pinned at two ends, separated by distance $l$. When $l$ is less than the beam length, the beam settles into one of the two symmetric equilibrium configurations. A sinusoidal force is applied at the ends, and the beam oscillates about the equilibrium position. As the excitation increases, however, the rod begins to snap back and forth between the two equilibria. If $w(x, t)$ is the lateral displacement,

$$
w(x, t)=\xi(t) \sin (\pi x) .
$$

For small forcing amplitudes, $\xi(t)$ is a periodic function; but as the forcing amplitude increases, $\xi(t)$ can become quite chaotic. The problem can be properly formulated mathematically, and simplified to a more familiar problem, the Duffing equation. The latter describes a simple nonlinear forced oscillator with frictional damping,

$$
\ddot{x}+\alpha \dot{x}+x+\beta x^{3}=f \cos (\omega t) \text {. }
$$

This example is sufficient to illustrate much of the phenomenology. When $\alpha=\beta=f=0,(6)$ corresponds to the equation of motion of a simple harmonic oscillator of unit mass and frequency. The motion in phase space is extremely simple: a circle in the $x \dot{x}$ plane, given by the parametric equations,

$$
x(t)=A \cos (\omega t+\phi) ; \dot{x}(t)=-A \sin (\omega t+\phi) .
$$

The motion is trivially regular. If damping is now introduced, keeping $\beta=f=0$, one has damped harmonic oscillations; the motion now consists of orbits that spiral exponentially into a sink at $\dot{x}=x=0$. When forcing is also included, one has a limit cycle behaviour, i.e., orbits converge asymptotically onto a limiting structure in the phase plane, and the motion is periodic on this limit cycle.

The sink and the limit cycle are examples of simple attractors. When nonlinearity is included in the equation of motion, it is possible to have more complicated attractorsin particular, strange, or chaotic, aperiodic attractors. One early example of such an attractor was found by Lorenz in a 3-mode truncation of the Navier-Stokes equation

$$
\begin{aligned}
& \dot{x}=10(y-x), \\
& \dot{y}=\mu x-y-x z, \\
& \dot{z}=x y-8 z / 3 .
\end{aligned}
$$

The strange attractor (SA) that occurs in this system is shown in perspective in figure 2 . The orbit spends random amounts of time on the two distinct portions of the attractor.

SA' $s$ are crucial in one picture of the onset of chaos and turbulence, the Ruelle-Takens model. Here chaos is presumed to occur by the creation of SA's, and the mechanism is as follows. Solutions to (1) are studied. For small $\mu$, some simple periodic motion is present; as $\mu$ increases, (Hopf) bifurcations occur; the motion is still regular, but now on $m$-dimensional tori, where $m>1$. When two such bifurcations have occurred, then it can be (mathematically) proven that any small perturbation suffices to create a SA, and hence chaos.

Currently, there exist several such scenarios for the onset of chaos and turbulence, each applicable in different circumstances. In the so-called Feigenbaum picture, we consider systems described by (1) or (2). As a function of the parameter $\mu$, the following behaviour pattern occurs. For $\mu<\mu_{0}$, there is some kind of a simple sink. At $\mu_{0}$, a bifurcation takes place and an orbit of period $\tau$ becomes stable and attracting. (Technically, this is through a "pitchfork" bifurcation). As $\mu$ is increased beyond a certain 


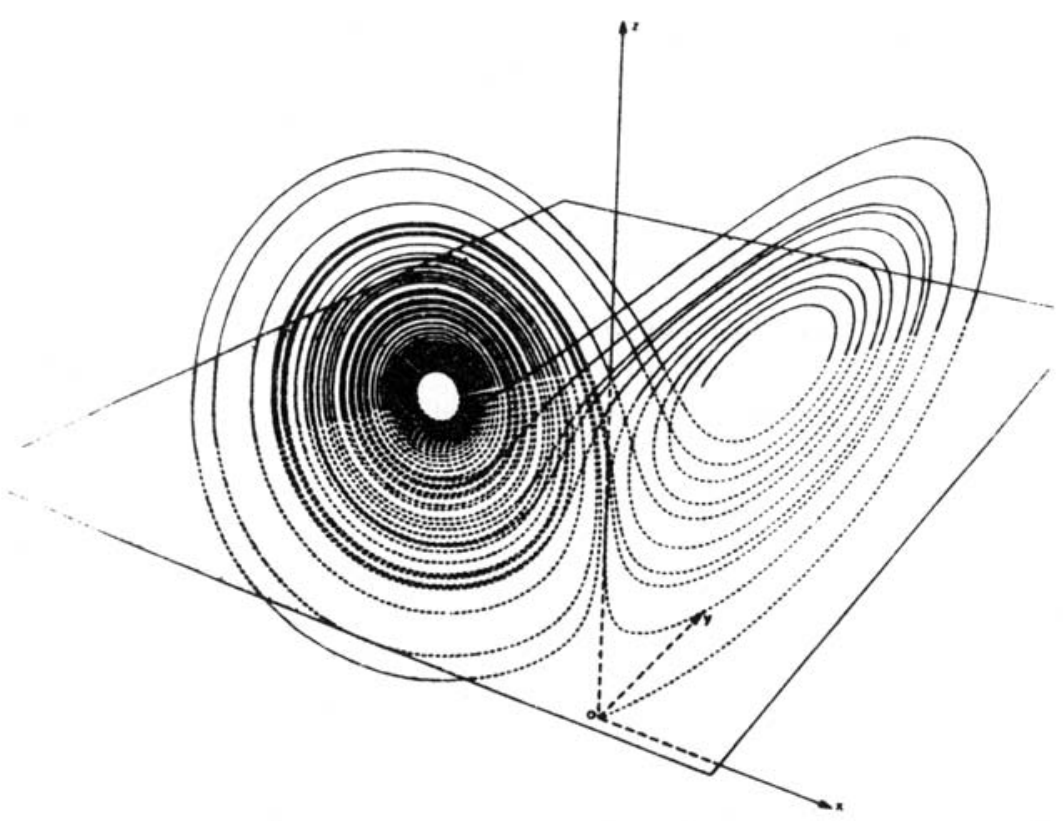

Figure 2. The strange attractor in the Lorenz system, equation (8), for $\mu=28$. The attractor consists of two almost planar portions, and the orbit wanders chaotically from one side to the other.

value $\mu_{1}$, the period $-\tau$ orbit loses stability, but one of period $2 \tau$ is born which is now stable and attracting. At a higher value $\mu_{2}$ another bifurcation takes place, with the period $-2 \tau$ orbit losing stability while a period $-4 \tau$ orbit becomes stable. This process repeats itself: at $\mu_{n}$, a period $-2^{n} \tau$ orbit becomes stable and attracting while the period$2^{n-1} \tau$ orbit loses stability. In the limit, one has a $2^{\infty} \tau$-period orbit, i.e., an orbit of infinite period, or chaos. The interesting thing is that $\mu_{\infty}$ is finite-the period-doubling cascade occurs quite rapidly, and furthermore, the rate of convergence of the bifurcation points,

$$
\delta_{n}=\frac{\mu_{n}-\mu_{n-1}}{\mu_{n+1}-\mu_{n}}
$$

logarithmically approaches $\delta=4.669201609 \ldots$ as $n \rightarrow \infty$. The most surprising fact is that the number $\delta$ is universal for a large general class of $F_{\mu}$. The precise form of $F_{\mu}$ does not matter (within certain restrictions). Wherever the period-doubling route to chaos is found, the number $\delta$ that one computes from measurement is identical to that found by Feigenbaum (i.e., 4.669 . . .). Historically, this number was discovered in a study of the discrete one-humped map of the unit interval known as the logistic equation, namely,

$$
\begin{aligned}
& x(j+1)=\mu x(j)[1-x(j)], \\
& 0 \leqslant x \leqslant 1,0 \leqslant \mu \leqslant 4 .
\end{aligned}
$$

(In fact, there are several other universal constants that can be found within such a picture.) The period-doubling route to chaos has been seen to occur in a wide variety of cases - in abstract maps such as (10), in differential equations such as (8), in a variety of 
theoretical experiments modelling chemical reaction kinetics, in forced oscillator problems, in a seven-mode truncation of the Navier-Stokes equation, and even in the equations that model repeated yielding in mechanical behaviour. Most crucially, though, a number of physical experiments have also confirmed this picture. The Rayleigh-Bénard flow problem mentioned earlier is one such. In this experiment, liquid helium is heated at around $4 \mathrm{~K}$, and the temperature at a point within the cell is monitored as a function of time. The Rayleigh number, $R$, plays the role of the parameter $\mu$. For low $R$, convective rolls are formed in the experimental cell; the motion is periodic. As the temperature is raised, the motion begins to become turbulent. The Fourier transform of the temperature as a function of the time clearly shows the existence of a basic frequency, $f$, and the subharmonic bifurcations, which give rise to peaks at $f / 2, f / 4, f / 8, f / 16$, etc. (see figure 3 ). The Feigenbaum constant computed from this experiment turns out to be about $4 \cdot 8$, amply confirming the general theory.

It must also be mentioned that the Rayleigh-Bénard experiment prepared differently displays the Ruelle-Takens behaviour as well. There is yet another common mechanism, intermittency, through which chaos can occur, via "tangent" bifurcations. Prior to the onset of full-scale chaotic behaviour, there are long periods of time when the motion is perfectly regular, interspersed with short bursts of erraticity. This route too can be observed experimentally.

In dissipative systems, chaos comes about in a manner different from that in conservative systems. Essential ingredients are bifurcations and attractors. One can have either period-doubling bifurcations and a periodic attractor in the Feigenbaum scenario (thus only one independent frequency), or bifurcations from a period orbit to tori, followed by a strange attractor in the Ruelle-Takens picture (thus at most three independent frequencies).

\section{Summary}

This talk has only briefly touched upon the basic phenomenology of irregular motion. Much of the interest in nonlinear dynamics arises from the existence of a large component of the unexpected and the counterintuitive. I have tried to outline the

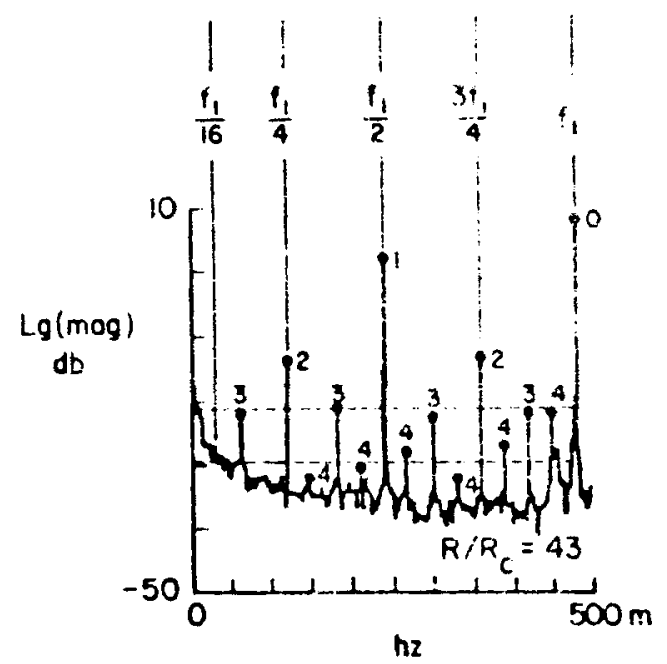

Figure 3. Period doubling subharmonics in the temperature power-spectrum of liquid helium in a Rayleigh-Benard experiment (from Maurer and Libchaber, loc. cit.). 
various paths or routes to chaos; unavoidably, there are gaps in this short presentation.

Although much progress has been made, there are still several open questions. For example, there is no minimal (or maximal) degree of complexity necessary to ensure chaos-very nonlinear systems can be integrable, while even the simplest (cubic) nonlinearity can induce chaos. And at the microscopic level, which may or may not be of interest here, quantum effects occur, and there is no clear understanding of how this can modify classical chaos.

When it does occur, chaos can have important consequences. For one thing, it becomes possible to apply statistical methods to advantage, if the behaviour is sufficiently random-like. On the other hand, chaos may be an undesirable feature if one wishes to remain in the region of the precisely predictable - this situation is common, for example, in particle storage rings where particle losses occur via erratic orbits: It is then essential to minimise or totally avoid chaos. The very ubiquity of chaos makes it necessary to examine dynamical processes in greater detail, whatever the context-this can well include mechanical behaviour.

\section{References}

\section{General references on chaos}

1980 Proc. Int. Conf. on Nonlinear Dynamics (ed) R H G Helleman (Ann. N.Y. Acad. Sci. No. 603)

Jorna S (ed) 1978 Topics in nonlinear dynamics (Am. Inst. Phys. Conf. Proc., Vol. 46)

Helleman R H G 1970 in F undamental problems in statistical mechanics (ed) E D G Cohen (New York: NorthHolland Publ) Vol 5, p 165

Moser J 1978 Math. Intell. 165

Ruelle D 1980 Math. Intell. 2126

May R M 1976 Nature (London) 261459

Hofstadter D 1981 Sci. Am. 24516

\section{Conservative systems}

Berry M V 1978 in Topics in nonlinear dynamics (ed) S Jorna Vol 46 (Am. Inst. Phys. Conf. Proc.) Ramaswamy R 1981 Phys. News 1260

\section{Dissipative systems}

Eckmann J P 1981 Rev. Mod. Phys. 53665

Kumar D 1982 Phys. News 13109

Tomita K 1982 Phys. Rep. 86113

Crutchfield J, Farmer J and Humberman B 1982 Fluctuations and simple chaotic dynamics Phys. Rep. 9245

\section{Some experimental references}

Maurer J and Libchaber A 1979 J. Phys. Lett. 40419

Maurer J and Libchaber A 1980 J. Phys. 41 C3

Feigenbaum M 1979 Phys. Lett. A74 375

Gollub J P, Besons S V and Steinmann J 1980 Proc. Int. Conf. nonlinear dynamics (ed) R H G Helleman (Ann. N.Y. Acad. Sci. No. 203)

\section{Other references}

Lorenz E N 1963 J. Atmos. Sci. 20130

Ruelle D and Takens F 1971 Commun. Math. Phys. 20167

Feigenbaum M 1978 J. Stat. Phys. 1925

Feigenbaum M 1979 J. Stat. Phys. 21669 


\section{Discussion}

G Ananthakrishna: Why is it often said that Landau's theory does not give a correct description of turbulence?

R Ramaswamy: The trouble with the Landau picture of turbulence is that as you vary a particular parameter, you have a series of bifurcations to the wrong geometrical object. These are in a certain sense bifurcations from a periodic orbit to a toroid, and thence a sequence of toroids. A limiting strange attractor does not appear.

G Ananthakrishna: Which route to the turbulent state is followed in the RayleighBenard case?

Ramaswamy: It has been shown that all 3 routes may be followed.

G Ananthakrishna: Is there any algorithm to get the Poincare map for multidimensional systems?

Ramaswamy: It is a difficult process.

S R Shenoy: Can one make a statement about the minimum order of the differential equations and of the nonlinearity in them in order to get chaos?

Ramaswamy: This is difficult. We do not even have a clear way of predicting whether a system is going to be integrable or not. A minimum requirement for chaos is of course nonlinearity, but it is not easy to make a more exact statement of general applicability.

K R Rao: Does the central peak in a second order phase transition have anything to do with chaos?

Ramaswamy: There is no analogy at all between the onset of chaos/turbulence with any kind of phase transition.

M Youssuff: How does one develop a probabilistic description of the chaotic state?

Ramaswamy: One of the reasons for studying chaos is to determine when ergodic theory is valid. Clearly, at the very least, chaos is needed for ergodicity to occur. 